\title{
Screening for Chronic Conditions Using a Patient Internet Portal: Recruitment for an Internet-based Primary Care Intervention
}

\author{
Suzanne G. Leveille, PhD, RN ${ }^{1,2}$, Annong Huang, $M D^{7}$, Stephanie B. Tsai, MA', \\ Saul N. Weingart, MD, PhD ${ }^{2,3}$, and Lisa I. lezzoni, MD, MSc ${ }^{1,2,4}$ \\ 'Division of General Medicine and Primary Care, Beth Israel Deaconess Medical Center, Brookline, MA, USA; ${ }^{2}$ Department of Medicine, \\ Harvard Medical School, Boston, MA, USA; ${ }^{3}$ Center for Patient Safety, Dana Farber Cancer Institute, Boston, MA, USA; ${ }^{4}$ Institute for Health \\ Policy, Massachusetts General Hospital, Boston, MA, USA.
}

BACKGROUND: Patient Internet portals have created new opportunities for assessment and management of chronic conditions.

OBJECTIVE: To conduct an online screening survey for a study recruitment using a secure patient Internet portal to identify primary care patients with untreated depression, chronic pain, or mobility difficulty before nonurgent office visits.

DESIGN: Internet-based screening survey for a randomized trial.

PARTICIPANTS: Patients who were registered portal users who had scheduled primary care appointments.

APPROACH: Electronic study invitations via the portal were sent to 4,047 patients with scheduled visits to 34 primary care physicians participating in the study. After clicking on a link in the study invitation, patients were consecutively shown the study description, consent form, and lastly, the screening survey to determine final eligibility for study participation.

RESULTS: Of the 2,113 (52\%) patients who opened the study invitation, 1,001 consented online to join the study and 981 (98\%) of these completed the screening survey. Of the respondents, 319 (33\%) screened positive for 1 or more of the 3 conditions.

CONCLUSIONS: The online screening survey conducted through the patient portal was effective in identifying patients with chronic conditions in advance of scheduled primary care visits for participation in an intervention study.

KEY WORDS: internet; ambulatory care; pain; screening; mobility. $J$ Gen Intern Med 23(4):472-5

DOI: $10.1007 / \mathrm{s} 11606-007-0443-6$

(c) Society of General Internal Medicine 2007

\section{INTRODUCTION}

Online patient-doctor communication creates new opportunities for improving clinical care. Given the brevity of primary care physician (PCP) office visits, patients and physicians face considerable challenges setting priorities to address clinical concerns. Information transmitted via secure patient Internet portals could allow identification of chronic problems before scheduled visits.

We developed an intervention conducted entirely through PatientSite, a secure patient Internet portal, to coach primary care patients before office visits about communicating with their PCPs regarding 3 common conditions: depression, chronic pain, and mobility difficulty. After providing informed consent online, patients completed an electronic survey screening for these conditions and assessing study eligibility. Participants randomized to the intervention received online coaching from experienced nurses regarding communicating effectively with their PCP about health concerns, specifically their screened condition(s).

This paper describes our experiences recruiting study participants online. Although others have explored the use of web-based screening tools, ${ }^{1-3}$ to our knowledge, this is the first report about using an Internet portal-based screening survey to identify patients with depression, chronic pain, or impaired mobility before scheduled PCP visits.

\section{METHODS}

PatientSite was developed to permit secure electronic patientprovider communication within Boston's Beth Israel Deaconess Medical Center (BIDMC) and affiliated practices. Patients logon to PatientSite from any computer using personal IDs and passwords. PatientSite allows e-mail messaging, prescription renewals, appointment and referral requests, and access to sections of online medical records. ${ }^{4}$ PCPs must first register on PatientSite before their patients can enroll. Patients access PatientSite most frequently to view laboratory or radiology reports and e-mail clinicians. ${ }^{4}$ To inform patients about new PatientSite messages, PatientSite sends e-mail messages to their external e-mail account saying they have new messages, along with a link to the PatientSite logon webpage. Of the estimated 80,000 patients within BIDMC and affiliates, 26,000 were registered PatientSite users when our project began in 2005.

\section{Participant Recruitment}

Our study first recruited PCPs registered on PatientSite from 2 hospital-based and 2 affiliated practices. Of 73 physicians, 57 met other eligibility requirements (electronic medical record 
use); 34 (60\%) consented, permitting us to invite their patients to join the trial.

We recruited patient participants by electronically scanning PCPs' appointment schedules for upcoming visits with patients registered on PatientSite. Identified patients automatically received invitations via PatientSite 4 weeks prior to their appointments. The e-mailed invitation listed "PatientSite" as the sender and "invitation to participate" as the subject line. The text indicated their PCP was participating in a study, briefly described the project, and provided an Internet link to a webpage containing more information. The system sent 2 electronic reminders to invitees not responding within 2 and 5 days, respectively, to the invitation. When invitees declined participation, the system stopped sending them messages. The study webpage contained an embedded link to an online consent form.

The consent form's first window contained a table of contents with 16 links allowing patients to select sections or scroll through the form. At the end of the form, patients could click buttons accepting or declining participation. Consenting patients were immediately shown the online screening survey and were sent a PatientSite message with a link to reaccess the screening survey if needed. BIDMC's Institutional Review Board approved all study procedures.

\section{Screening Procedures}

The screening survey included questions about demographics, general health, and targeted conditions: mobility difficulty, chronic pain, and depression (Fig. 1a). With skip patterns, the survey ranged from 25 to 52 items and required 6 to 7 minutes to complete, on average. Reports of any difficulty walking onehalf mile or climbing 10 steps without help from assistive devices or another person screened positive for mobility difficulties. ${ }^{5}$ This 2 -item mobility measure is strongly associ- ated with physical performance in older populations. ${ }^{6}$ The SF36 item regarding bodily pain in the past 4 weeks served as the initial screen for chronic musculoskeletal pain. ${ }^{7,8}$ This pain measure correlates well with other pain rating scales. ${ }^{9,10}$ When patients reported pain, present for $3+$ months in the past year, they were asked whether their pain primarily involved "joints, bones, muscles, or back," which confirmed a positive screen. Depression screening comprised a 2 -step process using the 8 item Patient Health Questionnaire (known as PH-8 or PRIMEMD). ${ }^{11,12}$ Persons with positive responses to the first 2 items were shown the remaining 6 PH- 8 items. We classified scores of 10 or higher, the recommended cutpoint, as positive for depression.

Persons who reported not receiving specialist care for their screened condition were eligible for the study and automatically randomized to treatment groups. Physicians received PatientSite messages indicating when their patients joined the study, specifying the screened condition(s). Intervention subjects received access to the intervention web site (Fig. 1b), automated nurse e-coaching messages via PatientSite to promote communicating with their $\mathrm{PCP}$, and individualized help planning their PCP visit. Control subjects received a message containing links to U.S. government web sites with general health information.

\section{Analysis}

Our focus here is the online recruitment and screening. We present descriptive statistics characterizing screening survey respondents. Chi-square tests compared persons who screened positive to those who screened negative for all 3 targeted conditions. We conducted a brief qualitative evaluation of all incidental (i.e., not specifically related to coaching interactions) e-mail correspondence sent to the study team by potential participants.

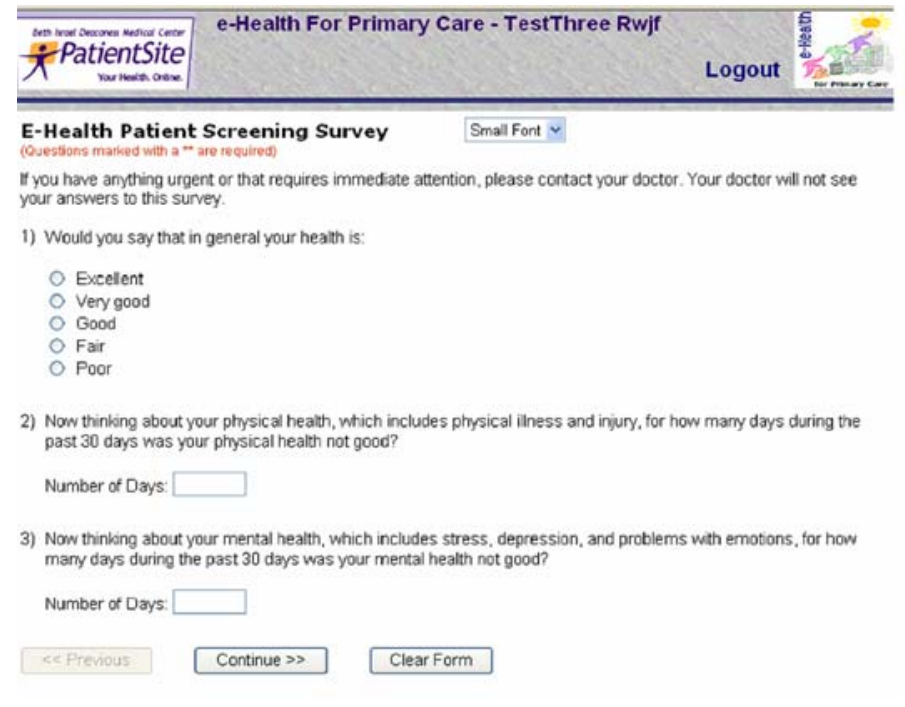

a

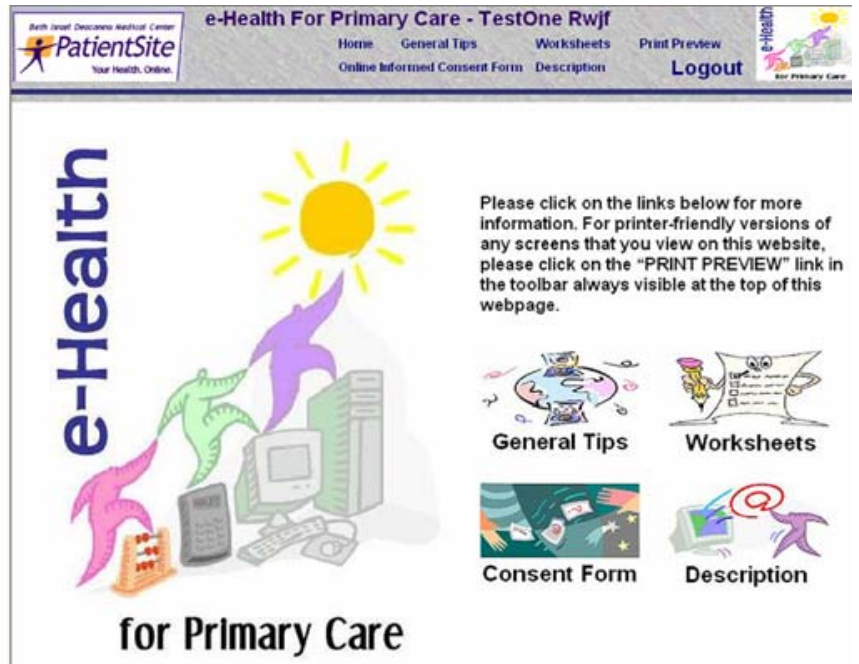

b

Figure 1. (a) E-Health study survey window. (b) E-Health study intervention webpage. Graphics used in the webpage were freely available from Microsoft Office Online. 


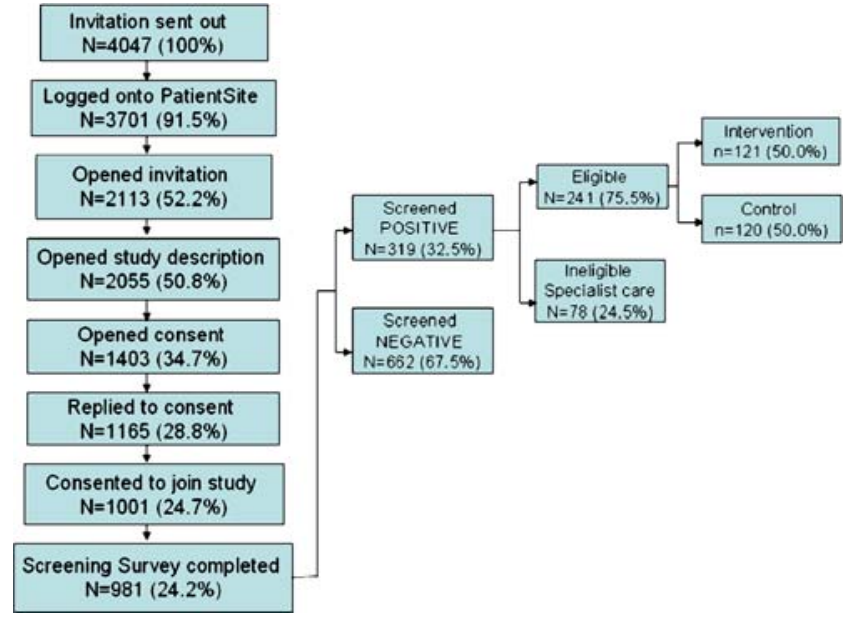

Figure 2. E-Health study recruitment flow chart.

\section{RESULTS}

From August 2005 to September 2006, the computerized system automatically e-mailed study invitations to 4,047 patients. Figure 2 shows screening and enrollment results, while Table 1 summarizes respondents' characteristics. The most common screened condition was chronic pain $(n=273$; $28 \%)$, followed by mobility difficulty $(n=93 ; 10 \%)$ and depression ( $n=56 ; 6 \%$ ); $25 \%$ ( 81 of 319 ) had 2 or more conditions. Patients screening positive were older and had less education than those screening negative. Survey respondents were older than BIDMC primary care patients in general $(81 \%$ vs $64 \%$ age $40+$, respectively); more were white ( $92 \%$ vs $65 \%$, respectively). Women comprised $61 \%$ of both groups.

Table 1. Baseline Characteristics of Survey Respondents of the E-Health Study

\begin{tabular}{|c|c|c|c|}
\hline Characteristics & $\begin{array}{l}\text { Screened } \\
\text { negative } \\
(n=662) \\
n(\%)^{*}\end{array}$ & $\begin{array}{l}\text { Screened } \\
\text { positive } \\
(n=319) \\
n(\%)^{*}\end{array}$ & $\begin{array}{l}\text { All respondents } \\
(n=981) n(\%)^{*}\end{array}$ \\
\hline \multicolumn{4}{|l|}{ Age (years) } \\
\hline $20-39$ & 141 (21.3) & $50(15.7)$ & 191 (19.5) \\
\hline $40-59$ & 394 (59.5) & $179(56.1)$ & $573(58.4)$ \\
\hline$\geq 60$ & 127 (19.2) & $90(28.2)$ & $217(22.1)$ \\
\hline Women & 404 (61.0) & 190 (59.6) & 594 (60.5) \\
\hline White race & 604 (91.9) & 285 (90.5) & 889 (91.5) \\
\hline \multicolumn{4}{|l|}{ Education } \\
\hline $\begin{array}{l}\text { High school } \\
\text { or less }\end{array}$ & $16(2.4)$ & $22(7.0)$ & 38 (3.9) \\
\hline $\begin{array}{l}\text { Not college } \\
\text { graduate }\end{array}$ & 88 (13.3) & 89 (28.2) & $177(18.1)$ \\
\hline $\begin{array}{l}\text { College } \\
\text { graduate }\end{array}$ & $556(84.2)$ & 205 (64.9) & 761 (78.0) \\
\hline $\begin{array}{l}\text { Married/ } \\
\text { partnered }\end{array}$ & $480(72.6)$ & $203(64.2)$ & 683 (69.9) \\
\hline Employed & 543 (82.5) & $213(67.2)$ & 756 (77.5) \\
\hline \multicolumn{4}{|l|}{ Self-rated health } \\
\hline $\begin{array}{l}\text { Good to } \\
\text { excellent }\end{array}$ & 629 (95.0) & 236 (74.0) & $865(88.2)$ \\
\hline Fair to poor & $33(5.0)$ & 83 (26.0) & 116 (11.8) \\
\hline
\end{tabular}

*Percents were based on nonmissing information. There were $<2 \%$ missing for all measures

\section{Patient Feedback and Technical Issues}

During the 1-year recruitment, we received 287 PatientSite messages from invitees, including several multiple e-mail exchanges with individuals. We received 121 messages from people replying to the invitation, either to decline or accept participation-unnecessary messages because the consent form contained buttons clearly marked "decline" and "accept." Thirteen messages expressed anger or annoyance about receiving study invitations. Patients reporting technical problems sent 74 messages. Although technical adjustments fixed most problems, we could not resolve some browser incompatibility difficulties: Macintosh computer users appeared to have more problems than PC users in accessing forms. Nonetheless, $8 \%$ of respondents used Macintosh computers. A dozen cases required telephone conversations to troubleshoot technical issues or resolve concerns about the informed consent.

In the final 3 months of recruitment, we added questions to the screening survey asking about experiences with online recruitment processes. Only 4 of 76 respondents reported technical difficulties. However, patients with significant technical problems may have been unable to complete these questions located at the end of the electronic form.

\section{DISCUSSION}

PatientSite, an Internet patient portal, served as a useful venue for recruiting and screening primary care patients for a randomized trial. More than half of invitees opened their emailed invitations-perhaps not surprising because, unlike ordinary e-mail, PatientSite inboxes almost exclusively involve communication between patients and providers. Some invitees may not have opened invitation messages because they assumed them to be standard appointment reminders. Nearly all patients (92\%) sent invitations logged onto PatientSite before their visits. An earlier PatientSite study indicated that patients use the portal for viewing laboratory and radiology reports more often than messaging. ${ }^{4}$

During early recruitment, direct telephone availability proved essential, primarily to give technical assistance. Telephone calls to study staff decreased markedly following initial technical adjustments. Many patients sent specific reply messages to invitation e-mails in addition to pressing automated enrollment buttons on the informed consent. The large number of messages accepting or declining participation may have occurred because the invitation message did not state that it was unnecessary to reply to the message. Also, the standard "reply" button at the top of each PatientSite message may have inadvertently encouraged replies.

This study had important limitations. We could not determine reasons invitees chose not to complete informed consents of screening surveys, such as not having - or fear of stigma for having - a screened condition. Our results suggest that many people were willing to report health problems in a portal-based screen. We would have expected that patients interested in previsit coaching might have more health problems (i.e., selection bias), but reliance on PatientSite may have introduced instead a healthy volunteer bias in our recruitment. ${ }^{4,13}$ Also, well-educated, Internet-savvy patients may have less need for coaching to promote productive interaction with their PCPs than persons with fewer personal resources. 
Future analyses will examine the outcomes of our intervention study. Findings presented here, however, suggest that screening patients through secure Internet portals might offer efficient and inexpensive ways to recruit patients for clinical research. Whether such screening could assist actual patient care - improving efficiency of primary care visits and helping address chronic concerns - remains unclear but merits future research.

Acknowledgments: This work was funded by the Robert Wood Johnson Foundation Health e-Technologies Initiative grant \# 051757. The authors wish to thank the following people for their valuable contributions to this research: Russell S. Phillips, Roger B. Davis, Linchang Huang, Qiang Wang, Marybeth Allen, and Melissa Cote.

Conflict of Interest: None disclosed.

Corresponding Author: Suzanne G. Leveille, $P h D, R N$; Division of General Medicine and Primary Care, Beth Israel Deaconess Medical Center, 1309 Beacon Street CO-219, Brookline, MA 02446, USA (e-mail: sleveill@bidmc.harvard.edu).

\section{REFERENCES}

1. Athale N, Sturley A, Skoczen S, Kavanaugh A, Lenert L. A webcompatible instrument for measuring self-reported disease activity in arthritis. J Rheumatol. 2004;31:223-8.
2. Farvolden P, McBride C, Bagby RM, Ravitz P. A Web-based screening instrument for depression and anxiety disorders in primary care. J Med Internet Res. 2003;5(3): 223.

3. Lin CC, Bai YM, Liu CY, et al. Web-based tools can be used reliably to detect patients with major depressive disorder and subsyndromal depressive symptoms. BMC Psychiatry. 2007;7:12.

4. Weingart SN, Rind $\mathbf{D}$, Tofias $\mathbf{Z}$, Sands $\mathbf{D Z}$. Who uses the patient internet portal? The PatientSite experience. J Am Med Inform Assoc. 2006; 13:91-5.

5. Rosow I, Breslau N. A Guttman health scale for the aged. J Gerontol. 1966;21:556-9.

6. Guralnik JM, Ferrucci L, Pieper CF, et al. Lower extremity function and subsequent disability: consistency across studies, predictive models, and value of gait speed alone compared with the short physical performance battery. J Gerontol. 2000;55:M221-31.

7. Stewart AL, Hays RD, Ware JE, Jr. The MOS short-form general health survey. Reliability and validity in a patient population. Med Care. 1988;26:724-35.

8. Ware J, Jr., Kosinski M, Keller SD. A 12-Item Short-Form Health Survey: construction of scales and preliminary tests of reliability and validity. Med Care. 1996;34:220-33.

9. Bostrom B, Sandh M, Lundberg D, Fridlund B. A comparison of pain and health-related quality of life between two groups of cancer patients with differing average levels of pain. J Clin Nurs. 2003;12:726-35.

10. Saal JA, Saal JS. Intradiscal electrothermal treatment for chronic discogenic low back pain: a prospective outcome study with minimum 1-year follow-up. Spine. 2000;25:2622-7.

11. Kroenke K, Spitzer RL, Williams JB. The PHQ-9: validity of a brief depression severity measure. J Gen Intern Med. 2001;16:606-13.

12. Kroenke K, Spitzer RL, Williams JB. The Patient Health Questionnaire-2: validity of a two-item depression screener. Med Care. 2003;41: 1284-92.

13. Hsu J, Huang $\mathbf{J}$, Kinsman $\mathbf{J}$, et al. Use of e-Health services between 1999 and 2002: a growing digital divide. J Am Med Inform Assoc. 2005; 12:164-71. 